\title{
Gewalt gegen ältere und pflegebedürftige Menschen
}

\section{Das Europäische Projekt MILCEA und seine Konsequenzen}

\section{UWE BRUCKER}

Uwe Brucker ist Fachgebietsleiter Pflegerische Versorgung beim Medizinischen Dienst des GKV-Spitzenverbandes (MDS) und Projektkoordinator von MILCEA

Gewalt gegen alte und pflegebedürftige Menschen ist in Deutschland ein marginalisiertes Randthema. In Ländern mit vergleichbarer Bevölkerungs- und Alternsstruktur ist die Prävention von Gewalt gegen alte und pflegebedürftige Menschen ein wichtiges Element einer demographiefesten Sozial- und Gesundheitspolitik. Was im Bereich der Kinder- und Jugendhilfe aber auch bei der Behindertenhilfe seit Jahrzehnten zur Selbstverständlichkeit geworden ist, stößt in Bezug auf alte Menschen noch immer an Tabugrenzen. Das Europäische Projekt MILCEA (Monitoring in Long-Term Care. Pilot Project on Elder Abuse) - wird vom MDS koordiniert und mit Mitteln der Europäischen Kommission gefördert. Projektpartner sind wissenschaftliche Institutionen in den Niederlanden, Luxemburg und Spanien sowie das Österreichische Rote Kreuz.

\section{Risikofaktoren für Gewalt bei Pflegebedürftigkeit}

Pflegebedürftigkeit geht einher mit einem Verlust an Eigenständigkeit und Selbstbestimmung; sowohl für den Gepflegten wie auch oft für den Pflegenden. Pflegebedürftigkeit bedeutet Abhängigkeit und führt zu einer sozialen Beziehung, die, von Machtgefälle geprägt und die höchst vulnerabel ist. Gelingt die Pflegebeziehung, wird auf die Würde, die Selbstbestimmung und die Unversehrheit des alten und pflegebedürftigen Menschen geachtet. Körperliche und psychische Gewalt, Vernachlässigung, finanzielle Ausbeutung, sexueller Missbrauch finden da keinen Platz. Und doch gibt es Gewalt, die meist im Verborgenen sowohl in familiären wie in außerfamiliären Pfle- gearrangements stattfindet gegen ältere pflegebedürftige Menschen. Die Gründe von Gewalt gegen ältere pflegebedürftige Menschen sind vielfältig. So kann es beispielsweise durch physische und psychische Belastungen in der Pflegesituation zu einer Überforderung der Pflegeperson kommen und in gewalttätigem Verhalten münden. Die Tendenz zu gewalttätigen Handlungen kann auch mit der Persönlichkeit und der Biographie des jeweiligen Täters zusammenhängen. Ein weiteres Risiko für Gewalt kann ein problematisches Verhältnis zwischen dem Pflegenden und der Pflegeperson sein. Auch eine Suchterkrankung (Drogen- oder Alkoholsucht) des Pflegenden erhöht das Risiko für eine Gewaltanwendung. Ein besonders hohes Risiko, Opfer von Gewalt zu werden haben Menschen mit Demenz und mit Behin- 
derungen und allgemein Personen, die in Abhängigkeit vom Hilfebedarf Dritter leben. Ein zusätzliches Risiko stellt dabei das Zusammenleben im gemeinsamen Haushalt dar. Die gelebte Beziehungsqualität zwischen Pflegendem und Gepflegten ist von entscheidender Bedeutung. Als weitere Risikofaktoren werden genannt die soziale Isolation, die Einkommensverhältnisse und die soziale Herkunft. Dabei spielen auch: soziale und kulturelle Normen (wie z. B. Altersdiskriminierung), die Tolerierung von Gewalt und Frauendiskriminierung eine Rolle.

Als Risiokofaktoren für Gewalt in stationären Pflegeeinrichtungen gelten für das dort beschäftigte Personal dessen schlechte Qualifikation, dessen alleine gelassen auf sich selbst gestelltes Arbeiten, die Gewalttoleranz im Team. Pflegepersonal, das Bewohnern von Pflegeheimen mit einem Mangel an Respekt begegnet und deren Selbständigkeit gering achtet, stellen ebenfalls ein höheres Risiko dar, dass Bewohner Opfer von Gewalt werden. Dem kann durch vielfältige Formen der Personalentwicklung und durch Personalmanagement entgegengewirkt werden wie die Reflektionsmöglichkeit über die eigene Arbeit (Supervision, kollegiale Beratung).

Die Unterstützung des Personals bei den Alltagsaktivitäten für die zu Pflegenden und die aktive Gestaltung der Beziehung ist ein Faktor, der gewaltmindernd wirken kann. Ebenso gelten die Vernetzung in der Kommune, der regelmäßige Besuch von Verwandten, Freunden und Nachbarn sowie eine positiv geprägte Lebenserfahrung und -einstellung als schützende Faktoren.

\section{Kaum Zahlen und dennoch Handlungsbedarf}

Die Prävalenzzahlen für Deutschland schwanken Görgen und Greve (2005) gehen von 1-10 Prozent Gewaltopfer bei über 65 Jährigen aus, der Bonner Gerontopsychiater Hirsch schätzt, dass 5-10 Prozent der älteren zu Hause versorgten Pflegebedürftigen und bis zu 20 Prozent in Pflegeheimen und mindestens jeder Zweite mit Demenz Gewalt ausgesetzt ist. Auch die Zahl der betreuungsgerichtlich genehmigten Fixierungen hat in Deutschland rasant zugenommen (mehr als 98000 neue Genehmigungen in 2010; eine Zunahme um 252 Prozent seit 1998). Hauptbetroffene: Menschen dieser "pflegerischen « Maßnahmen sind Menschen mit Demenz (Brucker 2011). Im Bereich der häuslichen Pflege geht man von einer hohen Dunkelziffer aus. Eine Befragung von pflegenden Angehörigen (Görgen 2010) ergab u. a.: »in den letzten 12 Monaten wurden vorgenommen 47,6\%: psychische Misshandlungen 19,4\% physische Misshandlung. Dabei wird auch von psychischen und physischen Übergriffe der zu Pflegenden berichtet.

In einer von der WHO 2011 vorgelegten Studie zum Thema wird von der Anwendung von körperlicher Gewalt: bei 4 Millionen (2,7\% >60 Jahre) Menschen im Jahr, von sexueller Gewalt: bei 1 Million (=0,7\%), von psychischer Gewalt bei 29 Millionen (=19,4\%) Menschen und von finanzieller Ausbeutung in 6 Millionen $(=3,8 \%)$ Fällen bei alten Menschen berichtet.

\section{In den europäischen Ländern gibt es bisher keinen systematischen Ansatz, um Gewalt gegen ältere Menschen in der Langzeitpflege verhindern zu können.}

Unumstritten ist die Notwendigkeit der Prävention. Dennoch gibt es in den europäischen Ländern bisher keinen systematischen Ansatz, um Gewalt gegen ältere Menschen in der Langzeitpflege identifizieren und verhindern zu können. An diesem Punkt knüpft das Projekt MILCEA $^{1}$ an. Übergeordnetes Ziel der Projektpartner ist es, Empfehlungen zu entwickeln, um ältere pflegebedürftige Menschen vor Gewalt schützen zu können. Diese Vorschläge sollten so gestaltet sein, dass sie die unterschiedlichen nationalen Gegebenheiten europäischer Länder in der Langzeitpflege berücksichtigen und somit von allen Ländern in Europa genutzt werden können.

Zu Beginn der Projektarbeiten musste zunächst definiert werden, was unter Gewalt gegen ältere Menschen speziell in der Langzeitpflege zu verstehen ist und wie man Gewalt oder ein Gewaltrisiko erkennen kann. Hierzu wurde eine Literaturanalyse durchgeführt. Ein Beirat aus wissenschaftlichen Experten und Praktikern der Langzeitpflege hat in jedem der Partnerländer die Arbeiten während der gesamten Projektlaufzeit begleitet.

Um Vorschläge zur Prävention von Gewalt gegen ältere pflegebedürftige Menschen entwickeln zu können, musste zunächst der Status Quo in den beteiligten Ländern untersucht werden. Hierfür wurden wichtige Institutionen in der Langzeitpflege in den einzelnen Ländern genauer unter die Lupe genommen. Als wichtig wurden diese eingestuft, wenn sie aufgrund ihrer Aufgabe im System der Langzeitpflege häufig mit älteren pflegbedürftigen Menschen in Kontakt kommen und somit das Potential haben, Gewalt zu erkennen und zu verhindern. Analysiert wurden u. a. die Aufgaben und die Rechtsgrundlagen dieser Akteure. Es wurde außerdem der Frage nachgegangen, ob diese Akteure bereits einen Auftrag haben, Gewalt zu erkennen und zu verhindern bzw. ob dieser Auftrag Teil ihres organisationalen Selbstverständnisses ist. Vertreter dieser Organisationen wurden dafür telefonisch $\mathrm{zu}$ folgenden Aspekten befragt: Sind die Organisationen überhaupt in der Lage, Gewalt oder ein Gewaltrisiko zu erkennen. Wie geschieht das? Werden spezifische Screening- oder Assessment-Instrumente eingesetzt? Was passiert, wenn der Verdacht von Gewalt vorliegt oder ein Risiko für Gewalt identifiziert worden ist? Gibt es bereits definierte Vorgehensweisen (z. B. auf nationaler oder regionaler Ebene) für solche Fälle? Wie sind die Organisationen miteinander vernetzt. Werden wichtige Informationen weitergegeben und an wen werden diese Informationen weitergegeben?

Anhand der Ergebnisse dieser Befragungen war es erstmals möglich, eine Bestandsaufnahme der Maßnahmen zur Gewaltprävention in den beteiligten Ländern vorzunehmen. Auf dieser Basis konnten in einem nächsten Schritt Stärken und Schwächen der Gewaltprävention in den jeweiligen Ländern identifiziert werden.

In keinem der beteiligten Länder gibt es eine Institution, die den unmittelbaren gesetzlichen Auftrag hat, Gewalt gegen ältere Menschen zu identifizieren und zu

1 www.milcea.eu 
verhindern. Zudem sind die Verantwortlichkeiten zwischen den Akteuren in der Langzeitpflege hinsichtlich der Prävention von Gewalt nicht klar definiert und kommuniziert. Pflegekräfte und andere Professionen in der Langzeitpflege sind weder ausreichend sensibilisiert für das Thema Gewalt, noch kennen sie wichtige Indikatoren und Risikofaktoren von Gewalt. In wenigen Ländern erst (wie z. B. Spanien) finden Screening-Tools, die der Identifikation von Gewalt gegen ältere Menschen dienen, Anwendung. In Deutschland ist dies beispielsweise nicht der Fall. Insgesamt sind in den Partnerländern die Strukturen, um Gewalt oder Gewaltrisiken in Pflegesituationen systematisch zu erkennen und $\mathrm{zu}$ erfassen nur schwach ausgeprägt.

Vor dem Aufbau von wirkungsvollen Strukturen zur Gewaltprävention bedarf es eines Bewusstwerdungsprozesses von allen unmittelbar und mittelbar in der Langzeitpflege tätigen Professionen, um sich der Relevanz des Themas Gewalt in der Langzeitpflege bewusst zu werden: Gewalt gegen ältere und pflegebedürftige Menschen darf nicht länger tabuisiert oder verharmlost werden. Erst wenn dieses Bewusstsein in allen Handlungsfeldern der Altenhilfe und -pflege gegeben ist, kann Gewalt oder ein Risiko für Gewalt erkannt und entsprechende Maßnahmen zum Schutz des pflegebedürftigen Menschen eingeleitet werden. Solange jedoch das Thema Gewalt in der Pflege nicht auf der politischen Agenda steht, wird ein solch systematischer Ansatz von Prävention nicht möglich sein. In den Ländern der EU müssen daher mehrere Voraussetzungen erfüllt sein: Daneben bedarf es umfassender Informations- und Fortbildungskampagnen zum Thema Gewalt in der Langzeitpflege für alle medizinischen, sozialen und pflegerischen Berufe. Unabdingbar ist nach Einschätzung der Projektpartner die Festlegung von Verantwortlichkeiten zur Prävention von Gewalt gegen Pflegebedürftige, um die zersplitterten Zuständigkeiten zu bündeln und Doppel- bzw- Mehrfachstrukturen zu vermeiden. Alle, die beruflich mit pflegebedürftigen alten Menschen arbeiten und die Pflegebedürftigen selbst müssen in Zukunft wissen, wer Ansprechpartner für Gewalt in der Pflege ist. In extremen Fällen, muss es auch - ähnlich wie bei misshandelten Frauen - rechtliche Mög- lichkeiten geben, den Täter vom Opfer fernzuhalten. Dafür sollten bei den Familiengerichten spezielle richterliche Zuständigkeiten für Gewalt geschaffen werden, damit zügig Abhilfe geschaffen werden kann.

Während in der professionellen Langzeitpflege Strukturen zur Gewaltprävention vorhanden sind, an denen die genannten Maßnahmen ansetzen können, stellt sich die Situation im häuslichen Bereich problematisch dar. Bei Personen, die ausschließlich durch Angehörige gepflegt werden, gibt es in der Regel kaum Kontakt zu Institutionen der professionellen Langzeitpflege. Hier nehmen Hausärzte eine wichtige Funktion in der Gewaltprävention ein.

\section{Es ist deshalb wichtig, dass sich Hausärzte ihrer herausgehobenen Rolle in der Gewaltprävention bewusst werden.}

Es ist deshalb wichtig, dass sich Hausärzte ihrer herausgehobenen Rolle in der Gewaltprävention bewusst werden. Sensibilisierungskampagnen zum Thema, Fortbildungen und die Schaffung einer breiten Fachöffentlichkeit versprechen mittelfristig Verbesserungen. Im informellen Bereich ist häufig die Belastung von Pflegenden Ursache für Gewalt. Hiergegen müssen die Beratungsdienste für Angehörige auf regionaler und lokaler Ebene stärker als bisher in das professionelle System der Langzeitpflege in zugehender Weise integriert werden.

\section{Konsequenzen aus diesem Projekt für Deutschland?}

Das Projekt MILCEA zeigt, dass die in der Langzeitpflege tätigen professionellen Akteure sich oftmals nicht über das Problem Gewalt gegen ältere Menschen bewusst sind. Und wenn dort ein Bewusstsein für das Thema besteht, ist es eher allgemein und abstrakt, die eigene Zuständigkeit oder Verantwortung wird nicht gesehen und das eigene Handlungsumfeld wird ausblendet. Das liegt u. a. daran, dass es auf der Systemebene weder verbindlich geregelte Zuständigkeiten im Falle von Gewalt existieren, noch vorliegende Instrumente zur Identifizierung von Gewalt oder einem Risiko Opfer von Gewalt bekannt und im routinemäßigen Einsatz sind.

Mit dem Verweis auf die bestehende Zuständigkeit der polizeilichen Vollzugsorgane wird auch ein darüber hinausgehender politischer Handlungsbedarf in Abrede gestellt. Dies verlagert das Problem Gewalt gegen ältere pflegebedürftige Menschen einseitig in ein primär Kriminologisches und vernachlässigt, die Ursachen von Gewalt, die vor allem in nicht funktionierenden sozialen Beziehungen und Strukturen $\mathrm{zu}$ suchen sind. Die Polizei ist für die Beendigung einer akut bestehenden Gewaltsituation ein wichtiger Akteur. Allerdings bereits bei der Frage, wie es danach weitergehen soll bei Opfer und Täter, sind soziale und sozialpsychologische Kompetenzen und $\mathrm{Zu}$ ständigkeiten gefragt. So gibt es in Deutschland keine Infrastruktur wie Akutbetten für Opfer von Gewalt aus Pflegebeziehungen (etwa analog den Frauenhäusern). Oder der Hinweis (entweder im Pflegegutachten oder im Bericht der Beratung nach $\$ 37$ Abs. 3 SGB XI), dass »die häusliche Pflege nicht in geeigneter Weise sichergestellt ist ", führt bei den Sachbearbeiters der Pflegekassen zu vielfältigen und heterogenen Reaktionen. Dieser Hinweis (häusliche Pflege nicht sichergestellt) kann auf den Risikofaktor für Gewalt in der Pflege hindeuten. Um das ausschließen zu können, bedarf es einer erneuten, fachlich und methodisch abgesicherten Beschäftigung mit diesem Pflegesetting. Doch dafür fehlen den Mitarbeitern der Pflegekassen nicht nur die bestehenden Instrumente, sondern auch regelmäßig verbindliche Verfahrensanweisungen, die Handlungssicherheit im weiteren Vorgehen geben.

In den Niederlanden hat die regierende Minderheitskoalition im März 2011 einen 10 Punkte-Maßnahmenplan »Senioren in sicheren Händen « veröffentlicht. Darin enthalten ist die Einrichtung einer landesweit einheitlichen NotrufHotline. In 35 festgelegten Kommunen sind Zufluchts- und Beratungszentren geschaffen worden, die für eine Region für Maßnahmen bei häuslicher Gewalt in Pflegesettings verantwortlich sind. Für dieses Jahr sind landesweite Aufklärungskampagnen geplant, ehrenamtliche Helfer werden geschult und für pro- 
fessionell Pflegende wird ab September 2012 ein E-Learning Programm zum Thema Gewalt gegen ältere und pflegebedürftige Menschen vorliegen.

In Deutschland fehlen für Gewaltsituationen gegenüber alten und pflegebedürftigen Menschen Zuständigkeitsregelungen wie sie z. B. im Kinder- und Jugendhilfesektor bestehen und wie sie gerade in den Niederlanden landesweit etabliert werden. Das MILCEA-Team im MDS will zur Weiterentwicklung dieser hierzulande nicht vorhandenen nationalen Infrastruktur weitere Vorschläge machen und diese auch erproben.

\section{Substitution ärztlicher Leistungen}

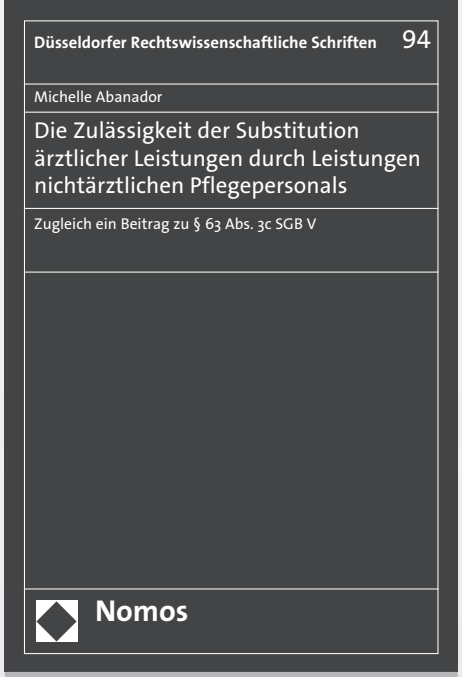

Das Werk erörtert, ob die Substitution ärztlicher Leistungen durch Leistungen nichtärztlichen Personals zulässig ist und welche Erfordernisse dafür erfüllt sein müssen. Die Verfasserin widmet sich dem Problem insbesondere im Hinblick auf § 63 Abs. 3c SGB V und gewährt einen Ausblick auf notwendige Kernelemente einer zukünftigen gesetzlichen Regelung.

Weitere Informationen: www.nomos-shop.de/13836

\section{Die Zulässigkeit der Substitution ärztlicher Leistungen durch Leistungen nichtärztlichen Pflegepersonals}

Zugleich ein Beitrag zu § 63 Abs. 3c SGB V

Von Dr. Michelle Abanador

2011, 234 S., brosch., 59,-€

ISBN 978-3-8329-6764-2

(Düsseldorfer Rechtswissen-

schaftliche Schriften, Bd. 94)

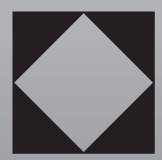

Nomos 